PALEO

Revue d'archéologie préhistorique

Numéro spécial | 2009-2010

Entre le marteau et l'enclume...

\title{
La percussion sur enclume en Italie centrale Tyrrhénienne
}

The bipolar-on-anvil percussion in central Thyrrenian Italy

Amilcare Bietti, Emanuele Cancellieri, Cinzia Corinaldesi, Stefano Grimaldi et Enza Spinapolice

\section{OpenEdition}

1 Journals

Édition électronique

URL : http://journals.openedition.org/paleo/1956

DOI : $10.4000 /$ paleo.1956

ISSN : 2101-0420

Éditeur

SAMRA

Édition imprimée

Pagination : 143-180

ISSN : 1145-3370

Référence électronique

Amilcare Bietti, Emanuele Cancellieri, Cinzia Corinaldesi, Stefano Grimaldi et Enza Spinapolice, « La percussion sur enclume en Italie centrale Tyrrhénienne », PALEO [En ligne], Numéro spécial |

2009-2010, mis en ligne le 25 avril 2012, consulté le 07 juillet 2020. URL : http://

journals.openedition.org/paleo/1956 ; DOl : https://doi.org/10.4000/paleo.1956

\section{(c) (i) $(9)$}

PALEO est mis à disposition selon les termes de la licence Creative Commons Attribution - Pas

d'Utilisation Commerciale - Pas de Modification 4.0 International. 


\title{
LA FRACTURATION EN SPLIT, UNE TECHNIQUE DE PRODUCTION DANS L'INDUSTRIE LITHIQUE DES TARES (Sourzac, Dordogne)
}

\author{
Jean-Philippe FAIVRE ${ }^{(1)}$, Jean-Michel GENESTE ${ }^{(2)}$ et Alain TURQ ${ }^{(3)}$
}

\begin{abstract}
Résumé : A partir du ré-examen de l'industrie du gisement des Tares et d'une expérimentation, une description de la fracturation en split est proposée. Elle se caractérise par une percussion rentrante, sur appui plus ou moins ferme, de direction strictement verticale, avec des percuteurs présentant une touche rectiligne.
\end{abstract}

Mots-clés : technologie lithique, Paléolithique inférieur et moyen, fracturation en split, touche rectiligne.

\begin{abstract}
Split fracture, a technique of production in the lithic industry from "Les Tares" (Sourzac, Dordogne). Following a re-examination of the lithic industry from Les Tares (Dordogne), as well as an experimental study, we propose a description of split fractures. They are produced by vertical (orthogonal) blows from inside the nodule, which is either rested on the ground or handheld. The hammer is characterised by a linear striking surface.
\end{abstract}

Key-words: lithic technology, Lower and Middle Paleolithic, split fracture, linear stricking surface.

\section{SITUATION GÉOGRAPHIQUE ET CHRONOLOGIQUE}

Situé en Dordogne, dans la partie basse de la vallée de l'Isle, en rive gauche près du village de Sourzac et à une vingtaine de kilomètres à l'ouest de Périgueux, le site des Tares est un gisement de plein air. II fut découvert fortuitement à l'occasion de travaux agricoles. Les récoltes initiales de vestiges lithiques abondants ont été suivies de plusieurs fouilles de sauvetage et d'une première interprétation des assemblages lithiques moustériens (Rigaud et Texier 1981). Les vestiges archéologiques se situent à environ trois mètres sous la surface du sol actuel et sont inclus dans des sédiments argilo-sableux interprétés comme des dépôts palustres (Bertran et Texier 1990) mis en place au cours d'une période antérieure au stade isotopique 6 , vraisemblablement lors d'une phase de transition entre un épisode interglaciaire et un épisode glaciaire (Texier, communication orale). Leur situation chronologique n'est actuellement pas connue avec précision (datations absolues en cours). Ils reposent sur les alluvions d'une terrasse de l'Isle cartographiée Fw2 (Texier 1982) et dont l'âge a été estimé à plus de $300 \mathrm{Ka}$ (Texier in Delpech et al.
1995). Ces différentes formations se situent à 65 mètres d'altitude absolue et sont étagées à une quinzaine de mètres au-dessus de l'étiage.

En 1991, les fouilles de sauvetage dirigées par J.-M. Geneste et J.-P. Texier ont permis de recueillir, sur $40 \mathrm{~m}^{2}$, un ensemble archéologique très peu dispersé sur le plan vertical $(20 \mathrm{~cm}$ maximum) composé de 6000 objets lithiques, dont 1043 objets retouchés, et de plusieurs centaines de vestiges paléontologiques attribuables à des équidés, bovidés et cervidés.

\section{ÉTUDE TECHNO-ÉCONOMIQUE DU SYSTÈME LITHIQUE GÉNÉRAL}

Dès la publication initiale de cet assemblage moustérien (Rigaud et Texier 1981), les auteurs en avaient signalé la spécificité et l'originalité. L'industrie fut alors rapprochée du Moustérien de type Charentien par son abondance de racloirs (plus de $55 \%$ ). Le fort taux de racloirs transversaux (12\%) et le caractère non Levallois du débitage avaient suscité une discussion par rapport au Moustérien Quina mais

(1) Université Bordeaux I, PACEA - De la Préhistoire à l'Actuel : Culture, Environnement, Anthropologie - UMR 5199 du CNRS, avenue des Facultés, 33405 Talence cedex - jeanphilippe.faivre@wanadoo.fr

(2) Centre National de Préhistoire, 24000 Périgueux, PACEA - Université Bordeaux I, UMR 5199 du CNRS, avenue des Facultés, 33405 Talence cedex - jean-michel.geneste@culture.gouv.fr

(3) Musée national de Préhistoire, 24620 Les Eyzies-de-Tayac-Sireuil, Université Bordeaux I, UMR 5199 du CNRS, avenue des Facultés, 33405 Talence cedex - alain.turq@ culture.gouv.fr 


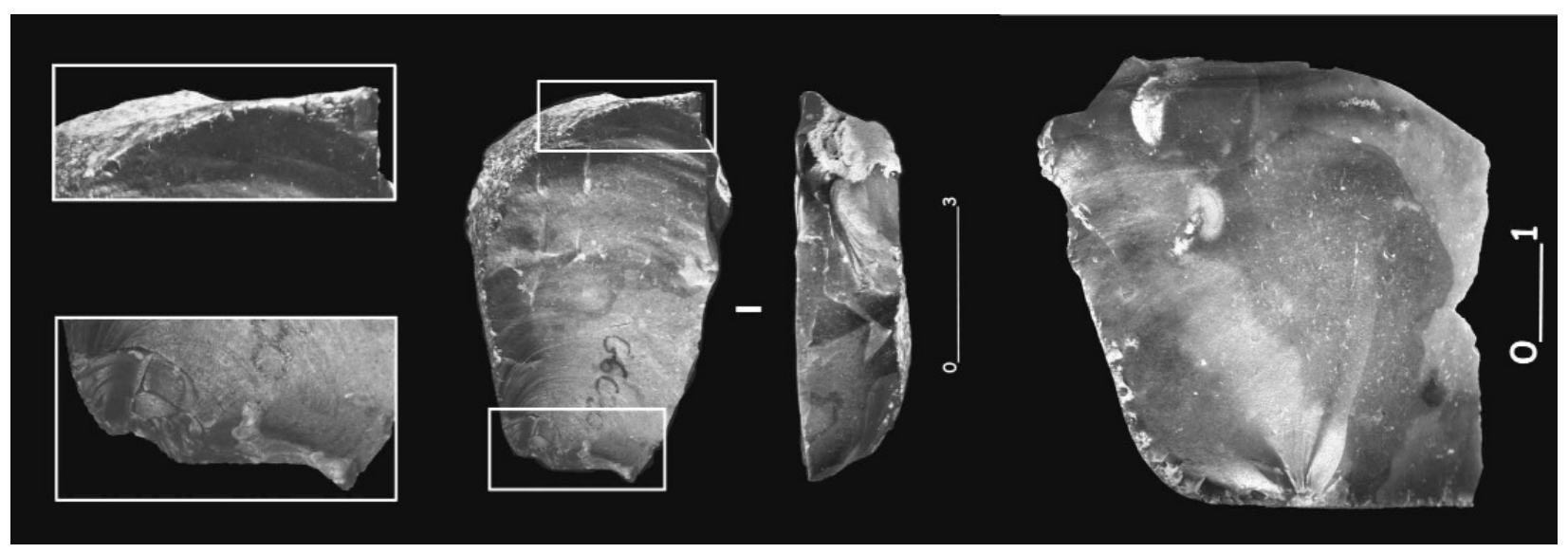

Figure 1 - Les Tares, commune de Sourzac, Dordogne : éclats à fracture en split, silex sénonien des alluvions de l'Isle.

Figure 1 - Les Tares (Sourzac, Dordogne) : flakes with « split » fracture, senonian flint from Isle valley.

également aux industries antéwürmiennes, notamment celles du sud de l'Angleterre (Highlodge), elles aussi caractérisées par un débitage d'éclats larges et épais au talon volumineux et au bulbe très convexe (op. cit.).

La chaîne opératoire de l'industrie des Tares présente, en outre, une structure ramifiée (Geneste 1991 ; Geneste et Plisson 1996 ; Bourguignon et al. 2004) : bon nombre de ces enlèvements ont été utilisés comme support de débitage (nucléus sur éclat) ou aménagés en outils à retouche écailleuse scalariforme. Certaines pièces retouchées, s'inscrivant dans un processus de gestion/réduction par réaffûtage, présentent le statut ambivalent d'outil-nucléus produisant de petits éclats à talon lisse incliné utilisés bruts ou retouchés.

\section{Plusieurs techniques de débitage}

L'analyse de l'industrie a initialement débouché sur la reconnaissance de deux techniques de débitage. La première est la percussion directe au percuteur de pierre dure sur la matière tenue par l'opérateur. Cette technique engage l'emploi d'une large gamme de percuteurs. Elle a pour but, en début de séquence opératoire, la production d'éclats massifs et larges, obtenus suivant une percussion rentrante verticale et, en fin de séquence, par des éclats de plus petites dimensions, de section et/ou de profil asymétrique (talon ou dos épais) obtenus par fracture conchoïdale.

La seconde technique, la percussion directe au percuteur dur de pierre sur la matière posée sur enclume (Geneste et Plisson, op. cit.) a été reconnue sur la base de la présence de nombreux enlèvements, essentiellement néo- corticaux, à surface d'éclatement en split (fig.1). Sur ces derniers, les stigmates reconnus comme inhérents à l'incidence du contrecoup sur l'enclume (Bordes 1947 ; Breuil et Lantier 1951 ; Kobayashi 1975 ; Sollberger et Patterson 1976 ; Mourre 1994, 1996 ; Kuijt et al. 1996 ; Gao 2000) sont rares ${ }^{4}$. De ce constat est née l'idée de l'emploi comme enclume de volumes de roches tendres telles des plaquettes de calcaires, découvertes à la fouille sous la forme de fragments.

Dès lors, la question qui découle de ce constat, et qui fait l'objet de cette contribution, s'attache à déterminer les différents paramètres techniques qui participent à la production d'enlèvements à surface de fracture en split. Pour cela, l'ensemble des éléments qui rentrent en jeu dans le cadre de la fracturation d'un volume de matière première sont ici pris en considération : nature des matériaux débités, type de percuteur (forme, poids, dureté), direction de la percussion, maintien du bloc débité lors de la taille (posé, calé, tenu dans la main).

\section{La fracture en split}

Près de $10 \%$ des enlèvements bruts de confection ou retouchés sont de grands éclats dont la surface d'éclatement s'avère correspondre à la définition de la fracture en split.

Bien que reconnue dans de nombreux ensembles lithiques et mentionnée à plusieurs reprises dans la littérature (Crabtree 1972) et enseignée par J. Pelegrin et P. J. Texier ${ }^{5}$, la fracture en split n'a pas, à ce jour, de définition précise établie sur la base des stigmates visibles sur les faces d'éclatement des enlèvements.

Comme dans tout mode d'action élémentaire sur la matière (Inizan et al. 1995), l'obtention des stigmates, récurrents, visibles sur la face d'éclatement, repose sur la relation binaire

(4) II convient de rappeler que ces stigmates techniques sont loin d'être systématiques lorsqu'il s'agit de galets de silex fendus sur enclume.

(5) Lors des stages de formation à l'étude de la technologie lithique et d'initiation à la taille organisé à Valbonne par le CNRS. 


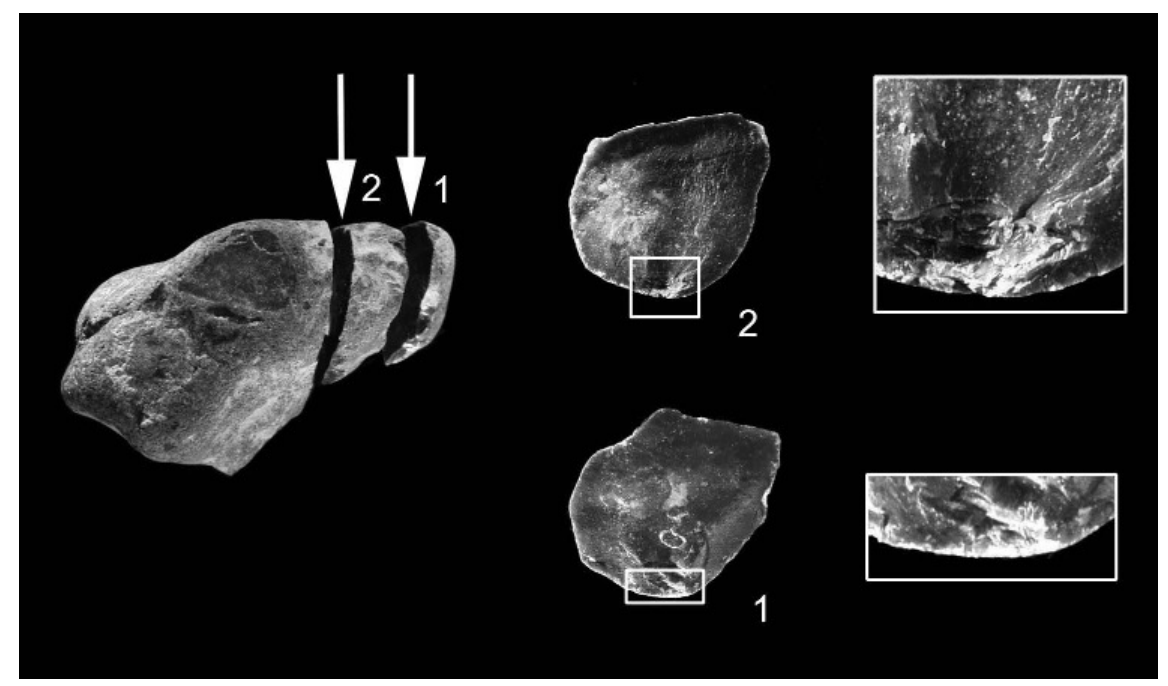

Figure 2 - Fractures en split produites expérimentalement, par percussion verticale, percuteur à touche rectiligne sur bloc posé au sol, sur silex sénonien des alluvions de la vallée de l'Isle.

Figure 2 - "Split" fractures experimentally produced by vertical percussion on blocks on ground, using pebbles as hammer with "straight touch". Senonian flint from Isle valley.

outil/gestes, chacun répondant à des critères de spécificité propres conditionnant le résultat.

La fracturation en split peut se définir de la façon suivante (fig. 2) : elle est produite par percussion rentrante, posée ou lancée, de direction strictement verticale et non tangentielle ${ }^{6}$. Son mécanisme se démarque ainsi de la fracture conchoïdale par un fendage dans l'axe de percussion sans incurvation de la trajectoire du percuteur.
Le plan de fracture est totalement plan : les surfaces positives et négatives ne présentent ni bulbe ni contre-bulbe. La zone d'impact est marquée par un écrasement caractéristique (compression) prolongé par des rides fines et serrées essentiellement concentrées dans le premier tiers de la face d'éclatement (phénomène mécanique). Les lancettes sont fortes et, lorsque la matière se prête à leur lisibilité, les ondulations sont marquées et parfaitement concentriques à partir de la zone d'impact.

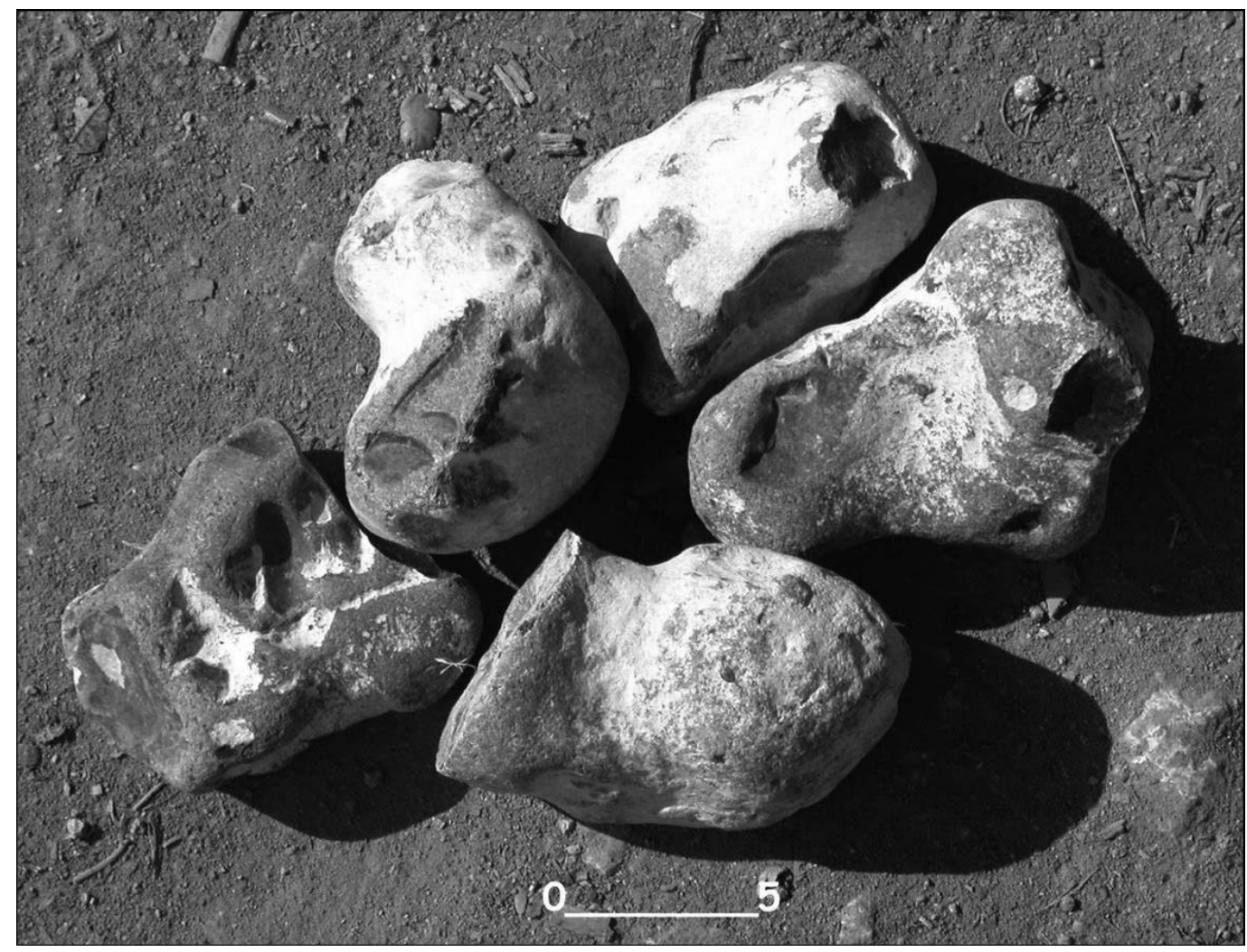

Figure 3 -

Nodules roulés et galets de silex sénonien de la vallée de l'Isle (échelle : 0 à $5 \mathrm{~cm}$ ) utilisés pour l'expérimentation.

Figure 3 - Rolled blocks and pebbles of senonian flint from Isle valley used for experimentation (size : 0 to $5 \mathrm{~cm}$ )

(6) Nous tenons vivement à remercier ici Jacques Pelegrin pour les longues discussions qui nous ont largement aidés lors de cette approche de la fracture en split. 

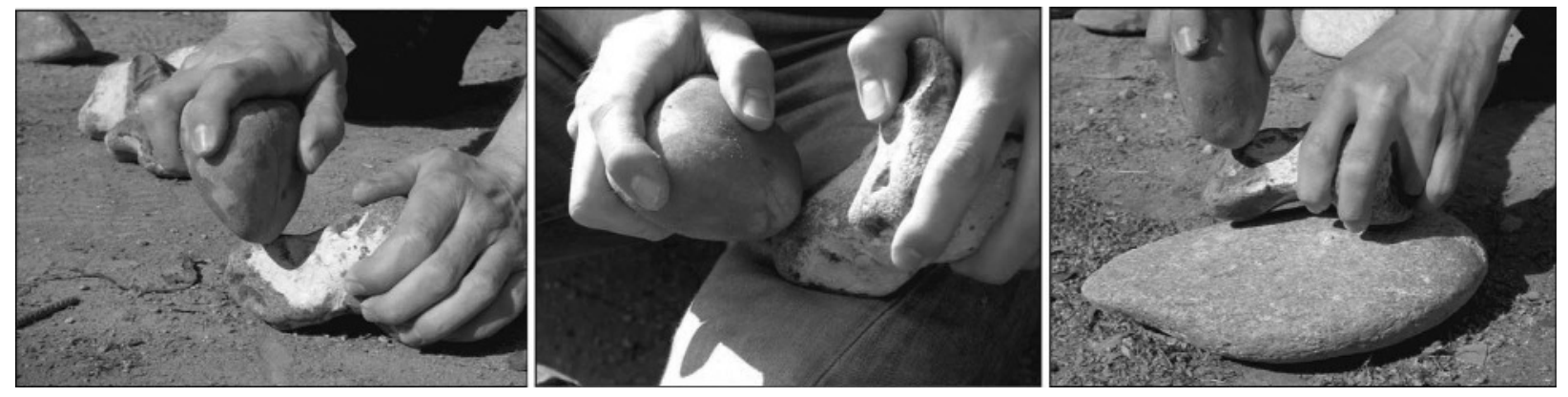

Figure 4 - Variantes de percussion directe testées expérimentalement : à gauche bloc posé au sol ; au centre bloc tenu en main ; à droite bloc posé sur enclume.

Figure 4 - Direct percussions experimentally tested : left, block on the ground; center, block held in hand; right, block resting on anvil.

Pour essayer de mieux appréhender les mécanismes techniques qui président au développement de ces stigmates, nous avons eu recours à l'expérimentation. Cette démarche a impliqué la recherche et l'utilisation de volumes de matières premières de même nature, tant au niveau de leur dimension que de leur source d'origine, que celles exploitées par les artisans des Tares. II s'agit de blocs de silex du Sénonien (principalement du Santonien de la région de Périgueux) que l'on trouve en abondance dans les formations alluviales de l'Isle. Ce sont des blocs de dimensions moyennes (inférieures à 15 centimètres), à surface d'altération néocorticale, aux formes contournées (fig. 3) et aux surfaces irrégulières opposant rarement des surfaces planes ou convexes.

\section{Techniques de percussion}

Trois types de percussion au percuteur dur, suivant une direction verticale, ont été testés : la percussion directe sur bloc posé, la percussion directe sur bloc tenu en main et enfin la percussion directe sur bloc posé sur enclume. Dans le cadre de l'application de la première technique, la nature du substrat sur lequel repose le bloc a varié quant à la dureté et à l'homogénéité (sol herbeux, terre damée, amas de débitage). Pour la troisième, une sélection de plusieurs matériaux, de nature pétrographique différente, ont été sélectionnés pour servir d'enclume (quartz, quartzite, basalte et calcaire). Par ailleurs, l'incidence de l'enclume, en terme de stigmates produits sur les enlèvements, diffère en fonction de la matière d'œuvre débitée. Si ces stigmates sont aujourd'hui bien connus et définis dans le cadre de l'application de cette technique à des matériaux de forte dureté, comme les galets de quartz et de quartzite de forme ovoïde ou quadrangulaire (Bracco 1993 ; Mourre 1996), ils restent sujets à une forte variabilité contextuelle pour les silex. La perception de l'implication éventuelle de la taille sur enclume dans l'industrie des Tares ne fait que justifier et renforcer la nécessité de l'emploi de matériaux identiques à ceux utilisés par les préhistoriques.

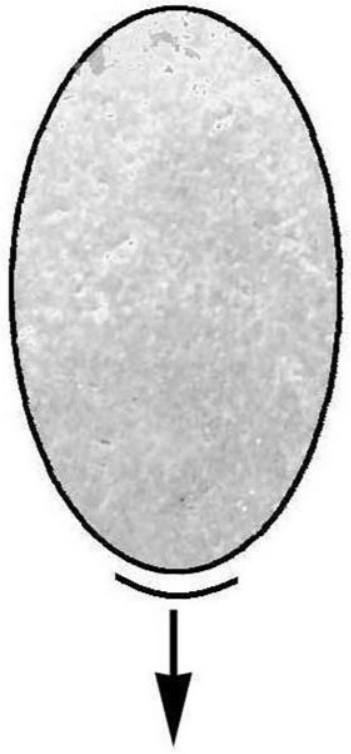

Percuteur à touche convexe

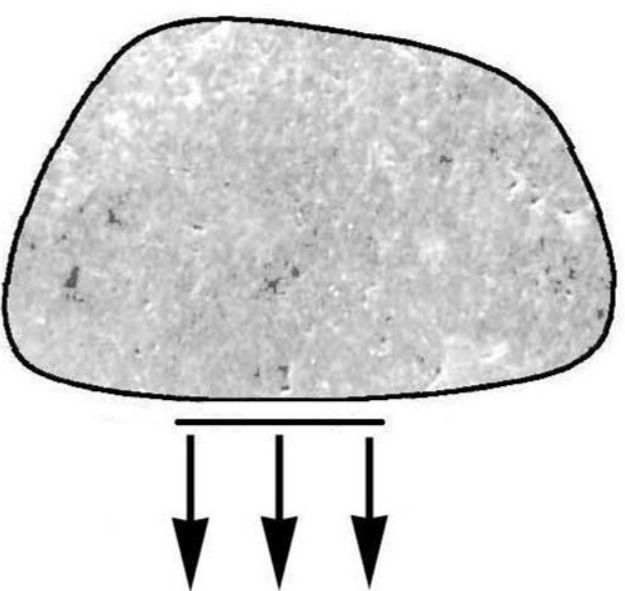

Percuteur à touche rectiligne
Figure 5 - Morphologie schématique des percuteurs expérimentaux.

Figure 5 - Schematic morphology of experimental hammers. 


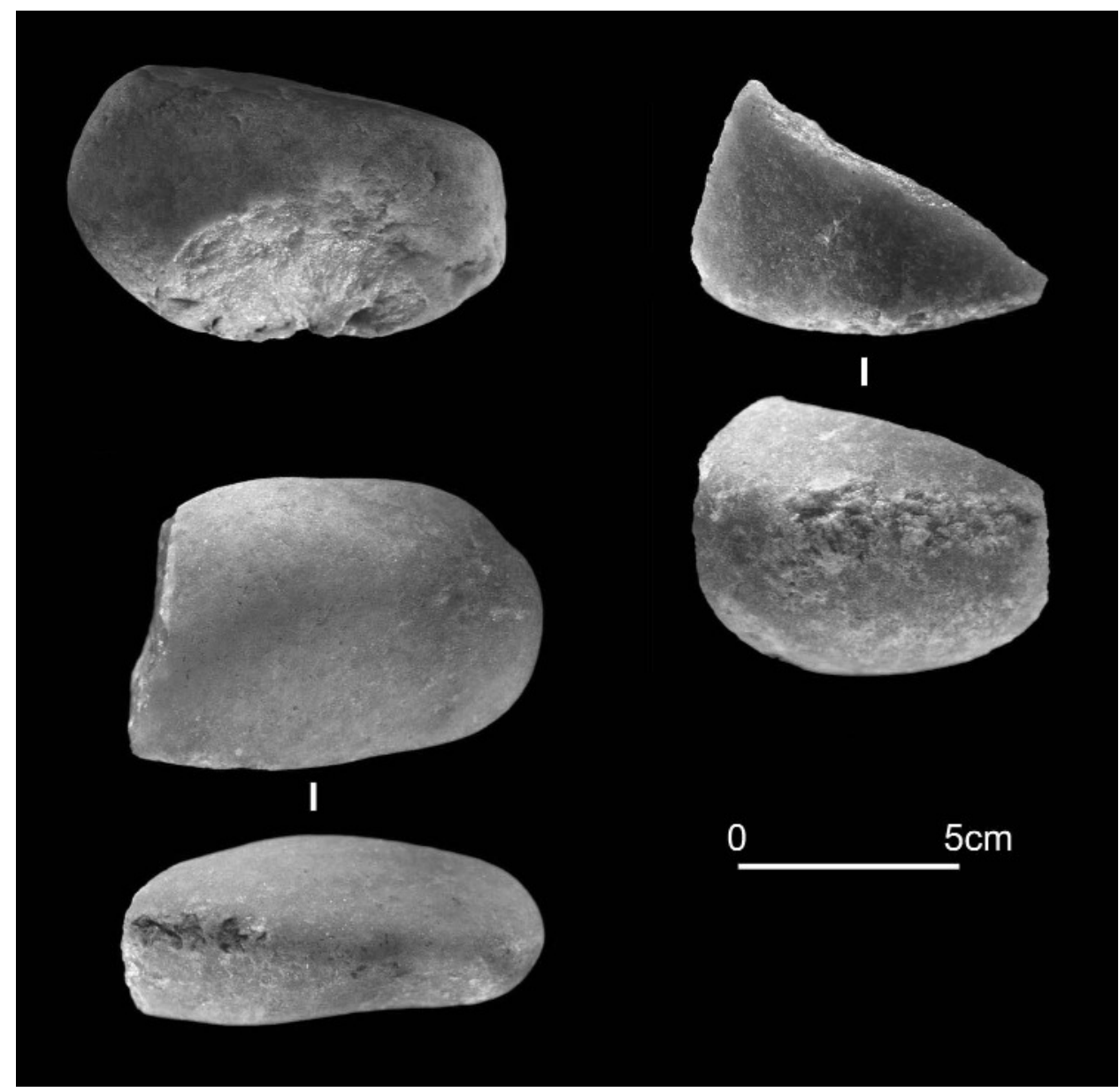

Figure 6 - Les Tares, commune de Sourzac,

Dordogne : percuteurs entiers ou fracturés à touche rectiligne, sur galets de quartzite des alluvions de la vallée de l'sle.

Figure 6 - Les Tares (Sourzac, Dordogne) : hammers whole or broken with "straight touch" on quartzite pebbles from Isle valley.

\section{Types de percuteur}

Un autre paramètre a concerné la sélection des percuteurs suivant des critères de forme, de volume, de dureté et de poids. Pour cela, une reconnaissance au sein de l'industrie de galets de quartzite présentant des plages d'écrasement ou de piquetage a orienté notre choix. A partir de la localisation de ces stigmates sur les pièces archéologiques, deux types de percuteur ont été sélectionnés (fig. 5) : les uns à zone de contact (touche) ponctuelle telle l'extrémité naturelle convexe des volumes, les autres présentant une touche rectiligne positionnée sur l'un des bords du galet et non à ses extrémités. Dans tous les cas, le poids des galets dépasse les 500 grammes.

\section{Principaux résultats}

Trois éléments nous paraissent importants dans le cadre de l'obtention de surface de fracture en split (tabl. 1) : la direction de percussion, l'appui du volume débité et la touche du percuteur.

L'obtention de bulbe de percussion semble, dans la plupart des cas, liée à un geste oblique réalisé avec un percuteur à touche convexe. Toutefois, un bulbe proéminent envahissant toute la surface d'éclatement apparaît fréquemment avec l'utilisation d'un percuteur à touche convexe dans le cadre d'une percussion verticale rentrante.

La fracture en split est, quant à elle, exclusivement produite avec un percuteur à touche rectiligne et suivant un geste vertical rentrant. Comme nous avons pu l'observer sur le matériel archéologique, cette fracture est systématiquement marquée par un écrasement linéaire au contact du percuteur avec le rognon débité. L'appui du bloc facilite la fracturation et (ou) favorise le contrôle du geste. Pour autant, un maintien dans la main de l'opérateur du volume débité, toujours suivant la même direction de percussion et la même touche, autorise le développement de ces plans de fracture (fig. 7). La production récurrente d'enlèvements à fracture en split n'est cependant réellement contrôlée que lorsque le volume débité est posé et calé (et ce quel que soit le substrat) pour permettre d'orienter et de maintenir favorablement le bloc. Ainsi, on peut observer une rotation du volume qui a pour but d'offrir le meilleur plan d'opposition entre le point d'appui ou calage et le plan de frappe.

Dans le cadre de l'utilisation expérimentale de l'enclume, les stigmates spécifiques lisibles en partie distale du support - signe d'appui axial ou non axial, présence de bulbe ou de contre bulbe, écrasement au niveau du point de contact avec l'enclume et détachement d'éclats parasites - 


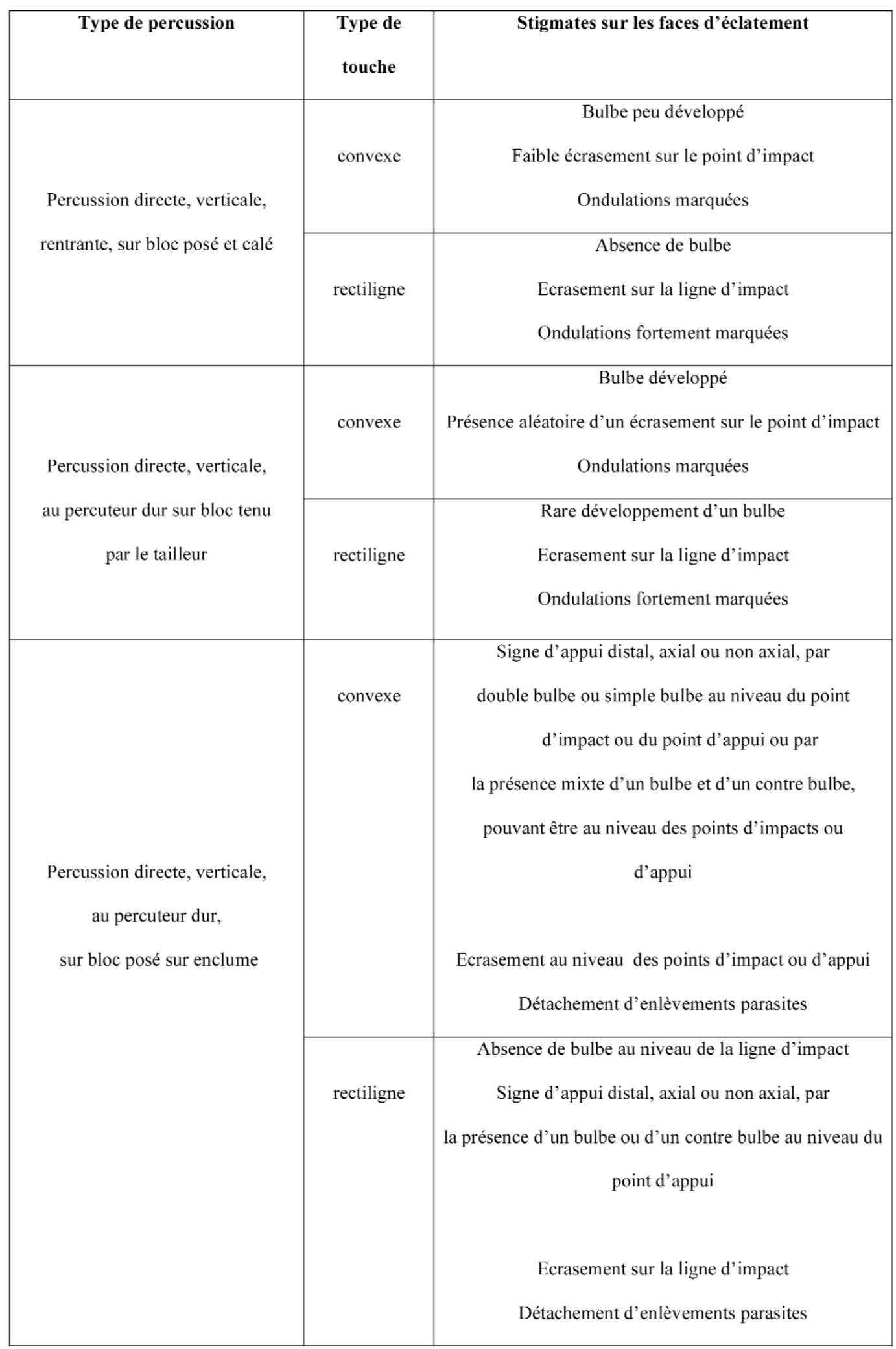

Tableau 1 - Résultats synthétiques de l'expérimentation des techniques de débitage.

Table 1 - Synthetic results of direct percussions experimentally used for flaking production. 


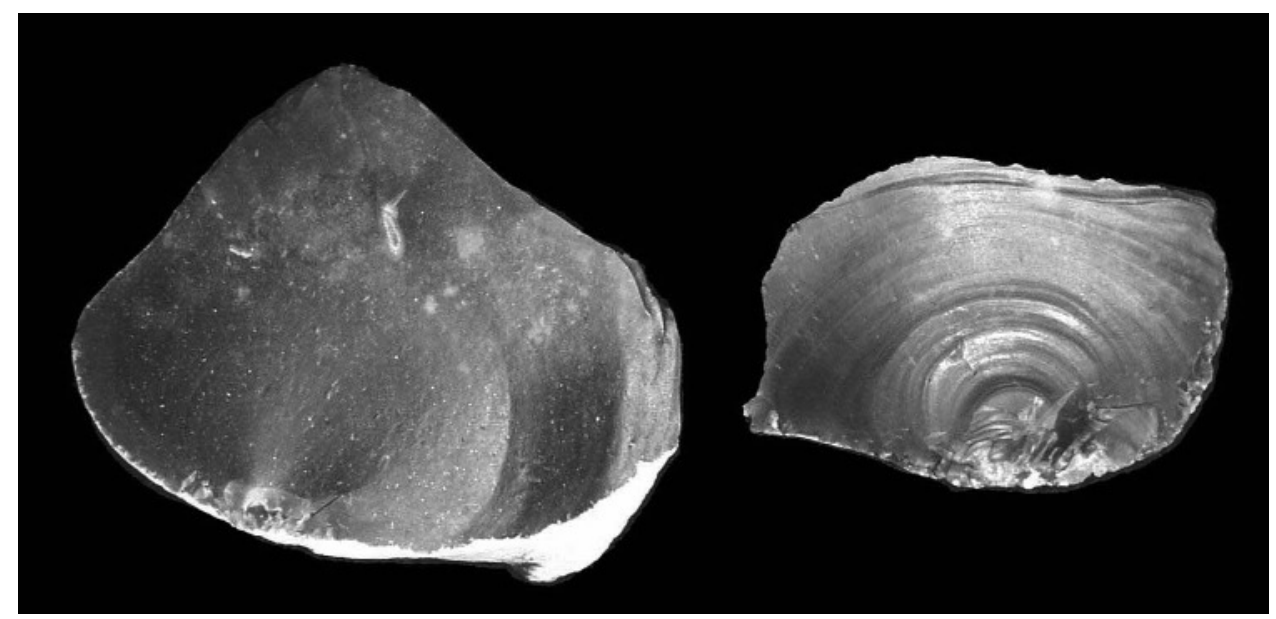

Figure 7 - Fractures en split expérimentales : percussion verticale rentrante, touche rectiligne, sur bloc tenu en main.

Figure 7 - Experimental "split" fractures : vertical percussion using an hammer with "straight touch" on block held in hand.

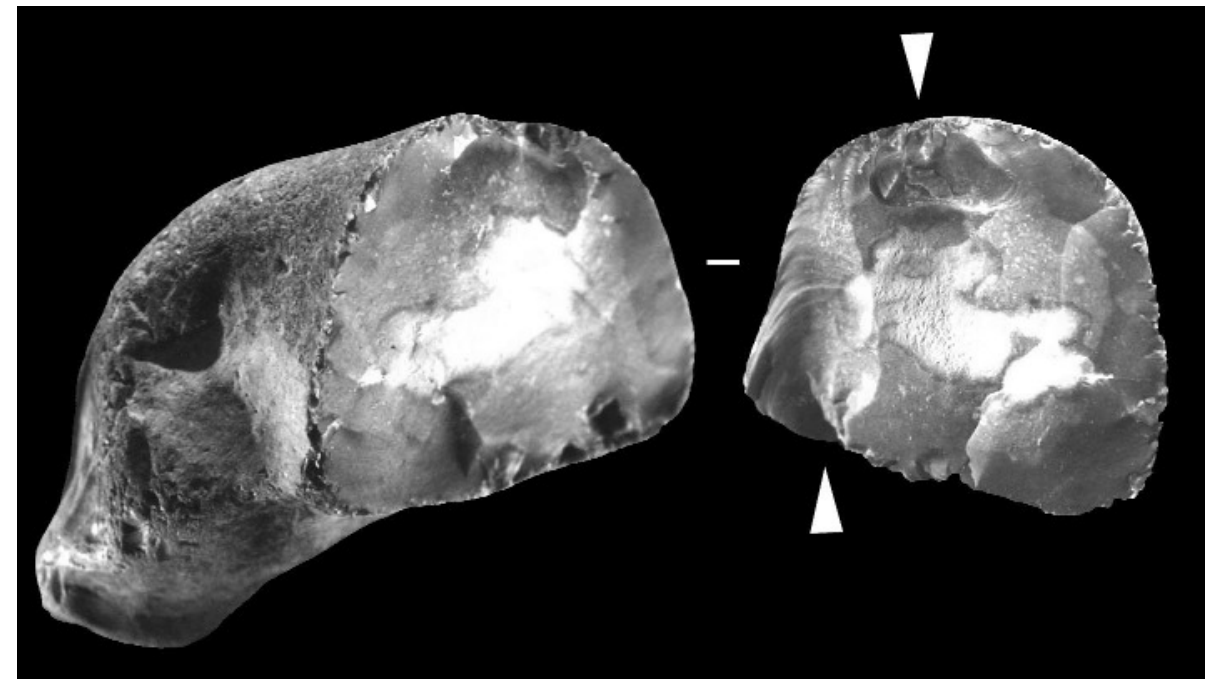

Figure 8 - Fracture en split expérimentale: percussion verticale sur enclume, touche rectiligne.

Figure 8 - Experimental «split» fracture : vertical percussion on anvil using an hammer with «straight touch».

sont généralement présents, indépendamment du geste et de la touche (fig. 8). II faut noter que les blocs de calcaire utilisés comme enclume se sont tous fracturés sous l'effet de la compression (écrasement) sans produire les effets du contrecoup escompté par l'utilisation de cette technique. Pour autant, ces matériaux ont constitué un substrat de calage tout à fait efficient.

\section{Les éclats à fracture en split des Tares : débitage sur enclume?}

La fonction principale de l'enclume consiste à stabiliser la pièce à fragmenter et de bénéficier d'un contrecoup « plus ou moins sec » (selon la dureté de la pièce dormante et sa surface en contact avec le sol). L'enclume, limitant le recul sous le choc de la percussion, augmente en apparence l'inertie de la pièce à fragmenter.

La rareté des stigmates distaux sur le matériel archéologique des Tares permet, sans totalement en remettre en question l'existence occasionnelle (pour ne pas dire fortuite), de discuter du rôle joué par la taille sur enclume dans cette industrie. Pour ce contexte, nous privilégions une stratégie qui consiste en un appui du bloc à débiter sur des substrats probablement diversifiés (sols, déchets de taille, plaquettes calcaires), ayant pour vocation de caler le volume. Cette stabilisation du volume débité, associant la main de l'opérateur au contact de calage, a une incidence sur la précision des gestes : positionnement adéquat du bloc pour offrir la meilleure surface de plan de frappe, contrôle de la trajectoire verticale du percuteur actif suivant des plans d'inclinaison variables. Le choix de la surface de plan de frappe semble être lié aux objectifs de compression visés par l'utilisation de la touche rectiligne sur le percuteur. Ceci se matérialise ici par une zone de contact plane ou faiblement convexe sur les surfaces de plan de frappe.

Un autre point est important à souligner. De nombreux enlèvements (éclats obtenus par fracture conchoïdale ou non) comportent sur leur face supérieure un ou plusieurs négatifs d'enlèvements à fracture en split. Souvent, ces négatifs sont adjacents à d'autres de profil concave d'inclinaison variables (sécants, subparallèles) et / ou à des plages néocorticales convexes dans le prolongement de leur partie distale. Cette dernière caractéristique parait en discordance avec la taille sur enclume puisque la présence de plage résiduelle néocorticale dans le prolongement distal des négatifs 

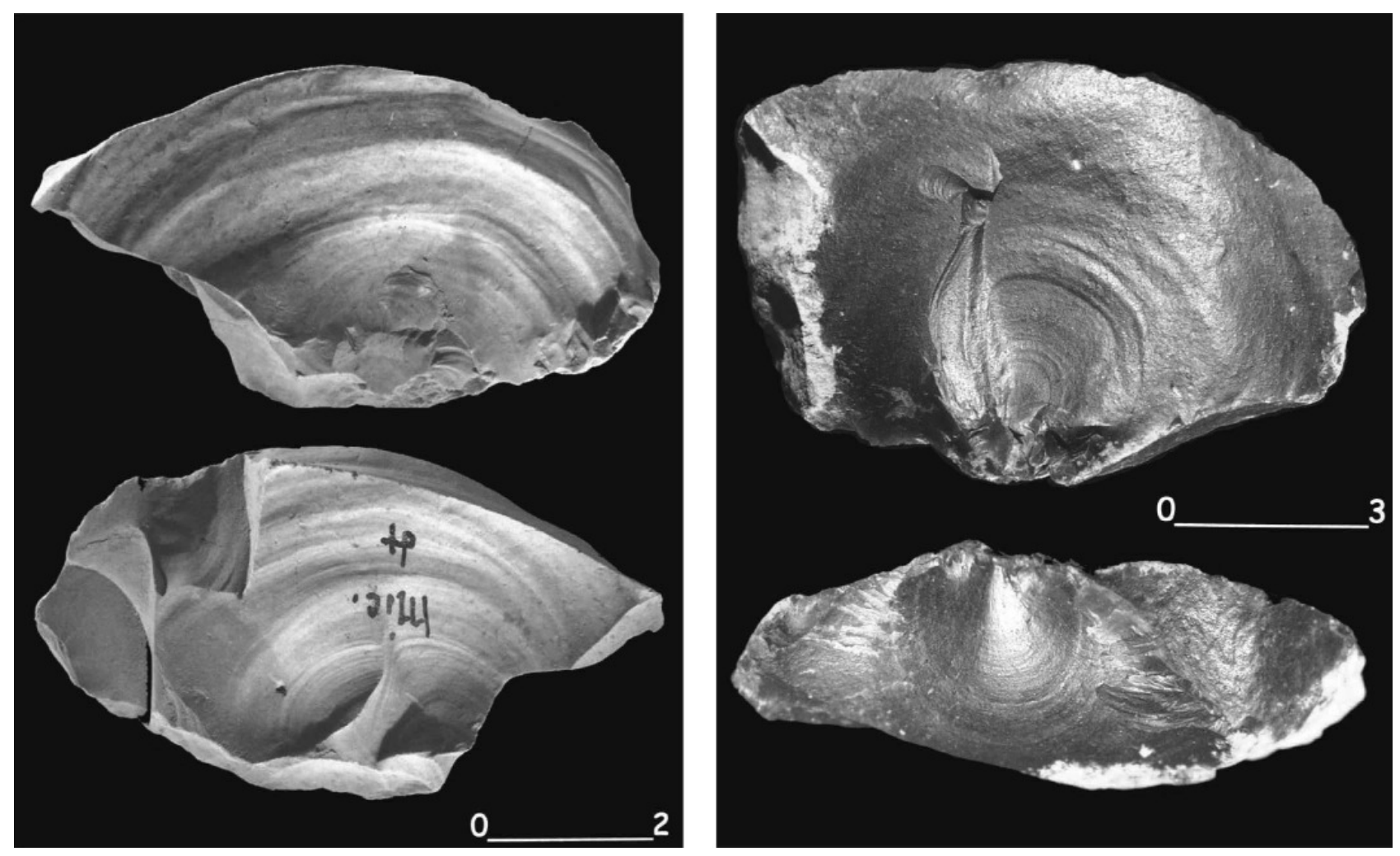

Figure 9 - Eclats à fracture en split : à gauche, la Micoque couche 4 fouilles D. Peyrony, éclat présentant deux surfaces de fracture en split ; à droite, Combe Grenal couche 22 fouilles F. Bordes.

Figure 9 - Flakes with "split" fracture : left, La Micoque, level 4 (excavation D. Peyrony), flake with two surfaces (upper and ventral) showing "split" fractures. Right, Combe-Grenal, level 22, Quina Mousterian (excavation F. Bordes).

en split rend compte de l'absence d'un contact opposé dur, axé ou désaxé, suivant une direction verticale. A l'inverse, cette particularité est tout à fait courante sur les produits résultant d'un bloc simplement posé et calé.

\section{CONCLUSION}

La fracturation en split est un mécanisme mettant en jeu plusieurs variantes. Sur le plan techno-économique, les enlèvements en split ne sont pas ici simplement caractéristiques de la phase d'amorçage du débitage puisque ces plans de fracture surviennent au cours de stades avancés de l'exploitation des blocs. De façon générale, ces éclats sont le reflet d'une démarche globale se fixant pour objectif la production de supports robustes, le plus souvent asymétrique en section ou en profil. La concrétisation de cette intention accorde un rôle décisif à la technique de percussion mise en œuvre. Celle-ci, directe et de trajectoire verticale rentrante, caractérise, pour la période abordée ici, bon nombre de productions lithiques du Paléolithique moyen (ancien et récent). C'est notamment le cas, pour ne citer que quelques exemples, des industries tayaciennes de la Micoque et des industries moustériennes de type Quina de Combe-Grenal (fig. 9) dans lesquelles la percussion directe verticale rentrante détermine des enlèvements à plan de fracture en split. Rares dans les contextes Quina, les enlèvements présentant ces plans de fracture spécifiques sont beaucoup plus nombreux dans les industries de la Micoque ${ }^{7}$. II est intéressant de souligner que les mêmes stigmates sont présents dans les déchets des ateliers de fabrication de meules en silex. L'étude des chaînes opératoires a montré que là aussi, gestes, appui du volume débité et touche rectiligne de l'outil en fer, en sont directement à l'origine (Turq 2003 et ce volume).

\section{BIBLIOGRAPHIE}

BERTRAN P., TEXIER J.-P. 1990 - L'enregistrement des phénomènes pédosédimentaires et climatiques dans les dépôts colluviaux d'Aquitaine: l'exemple de la coupe des Tares. In: Méthodes et concepts en stratigraphie du Quaternaire européen. Actes du Colloque international, Dijon 5-7 décembre 1988. Quaternaire, 1, n. 1, p. 77-90.

(7) Les séries de la Micoque mentionnées ici correspondent à celles provenant des niveaux 3 et 4 , fouillés par D. Peyrony, conservées au Musée national de Préhistoire. 
BORDES F. 1947 - Etude comparative des différentes techniques de taille du silex et des roches dures. L'Anthropologie, t. 51, n 1-2, p. 1-29, 13 fig.

BOURGUIGNON L., FAIVRE J.-Ph. et TURQ A. 2004 Ramification des chaînes opératoires : une spécificité du Moustérien ? Paléo $n^{\circ} 16$, p. 37-48

BRACCO J.-P. 1993 - Mise en évidence d'une technique spécifique pour le débitage du quartz dans le gisement badegoulien de la Roche à Tavernat (Massif Central, France). Préhistoire Anthropologie Méditerranéenne, 2, p.43-50.

BREUIL H., LANTIER R. 1951 - Les Hommes de la pierre ancienne - Paléolithique et Mésolithique. Paris : Payot, 335 p.

CRABTREE D.-E. 1972 - An introduction to flintworking (Technology, Glossary. Hors série 28, Musée et Université de l'Etat d'Idakho.

DELPECH F., GENESTE J.-M., RIGAUD J.-Ph. et TEXIER J.-P. 1995 - Les industries antérieures à la dernière glaciation en Aquitaine septentrionale : chronologie, paléoenvironnements, technologie, typologie et économie de subsistance. Paléo, supplément 1, actes du colloque de Miskolc, juin 1995, p. 133-163, ill.

GAO X. 2000 - Core reduction at Zhoukoudian locality 15. Archaeology, ethnology \& anthropology of Eurasia, $\mathrm{n}^{\circ} 3$, 3, p. 2-12.

GENESTE J.-M. 1991 - Systèmes techniques de production lithique : variations techno-économiques dans les processus de réalisation des outillages paléolithiques. Techniques et culture, 17-18, 1991, p. 1-35.

GENESTE J.-M., PLISSON H. 1996 - Production et utilisation de l'outillage lithique dans le Moustérien du SudOuest de la France: les Tares à Sourzac, Vallée de l'Isle, Dordogne. In : Proceedings of the International Round Table: Reduction processes (" chaînes opératoires ») for the European Mousterian, Bietti, A. et Grimaldi, S., Eds. Rome: Quaternaria Nova VI, p. 343-367.
INIZAN M-L, REDURON-BALLINGER M., ROCHE H. et TIXIER J. 1995 - Préhistoire de la pierre taillée $t$. 4 Technologie de la pierre taillée. Meudon : CREP, $199 \mathrm{p}$.

KOBAYASHI H. 1975 - The experimental study of bipolar flakes. In : Lithic Technology - Making and using stone tools, Swanson, E., Ed. The Hague: Mouton, p. 115-128.

KUIJT L., PRENTISS W.c. \& POKOTYLO D.L. 1996 Bipolar reduction : an experimental study of debitage variability. Lithic Technology, vol. 20, n 2, p. 116-127.

MOURRE V. 1994 - Les industries en quartz au Paléolithique moyen. Approche technologique de séries du Sud-Ouest de la France. Mém. de maîtrise Univ. de Paris X, UFR SSA, Département d'Ethn. Sociol. comparative et de Préhist. 2 vol., 111, 84.

MOURRE V. 1996 - Le débitage sur enclume au Paléolithique inférieur et moyen. Techniques, méthodes et schémas conceptuels, Université de Paris X - Nanterre, Mémoire de DEA, $45 \mathrm{p}$.

RIGAUD J.-Ph., TEXIER J.-P. 1981 - A propos des particularités techniques et typologiques du gisement des Tares, commune de Sourzac (Dordogne). Bulletin de la Soc. préhist. franç., 78, n. 4, 109-117.

SOLLBERGER J., PATTERSON L. 1976 - The myth of bipolar flaking industries. Newsletter of Lithic Technology, vol.V, $n^{\circ} 3$, p. 40-41.

TEXIER J.-P. 1982 - Les formations superficielles du bassin de l'Isle. Paris, CNRS. Centre de publication de Bordeaux, 316. Cahiers du Quaternaire, 4.

TURQ A. 2003 - De la matière première lithique brute à la mise au jour de l'objet archéologique : propositions pour une meilleure exploitation du potentiel informatif du matériel lithique illustrées par quelques exemples du Paléolithique aquitain. Perpignan : Université de Perpignan, mémoire original pour l'obtention d'une habilitation à diriger des recherches, 165 p., CV et travaux (3 volumes). 
